\title{
32.
}

\section{Développement d'une formule qui donne en même temps les nombres de Bernoulli et les coëfficients de la série qui exprime la sécante.}

(Par Mr. O. Schlömilch, prof. à l'université de Jena.)

Dans le $4^{\text {me }}$ tome de ce journal, Mr. Scherk a démontré une fọrmule qui donne immédiatement les coefficients $\boldsymbol{B}_{0}, \boldsymbol{B}_{2}, \boldsymbol{B}_{4}$ etc. de la série

$$
\sec x=B_{0}+\frac{B_{2}}{1 \cdot 2} x^{2}+\frac{B_{4}}{1 \cdot 2 \cdot 3 \cdot 4} x^{4}+\ldots
$$

et ceux $B_{1}, B_{3}, B_{5}$ etc. de la série

$$
\operatorname{tang} x=\frac{2^{2}\left(2^{2}-1\right)}{1 \cdot 2} B_{1} x+\frac{2^{4}\left(2^{4}-1\right)}{1 \cdot 2 \cdot 3 \cdot 4} B_{3} x^{3}+\ldots
$$

Les derniers sont les nombres de Bernoulli. L'auteur a dévéloppé la dérivée

$$
\frac{\partial^{n} \operatorname{tang}\left(\frac{1}{4} n+\frac{1}{2} x\right)}{\partial x^{n}}
$$

par laquelle on obtient pour $x=0$ une formule qui donne $B_{n}$, quel que soit l'indice. Il tire cette dérivée d'un théorème de Laplace relatif à la dérivée

$$
\frac{\partial^{n}}{\partial h^{n}}\left\{\frac{1}{e^{h}+1}\right\}
$$

en $y$ supposant $\boldsymbol{y}$ imaginaire.

On peut doriner une autre forme à ce calcul, en fesant usage de la formule générale

1. $\frac{\partial^{n} f\left(e^{x}\right)}{\partial x^{n}}=\frac{1}{1} \stackrel{n}{K}_{1} e^{x} f^{\prime}\left(e^{x}\right)+\frac{1}{1 \cdot 2} \stackrel{n}{K}_{2} e^{2 x} f^{\prime \prime}\left(e^{x}\right)+\ldots+\frac{1}{1 \cdot 2 \cdots n} \stackrel{n}{K}_{n} e^{n x} f^{(n)}\left(e^{x}\right)$, dont les coëfficients $K_{1}, K_{2}$ etc. sont déterminés par l'équation

$$
\text { 2. } \quad \stackrel{n}{K}_{p}=p^{n}-(p-1)^{n} p_{1}+(p-2)^{n} p_{2}-(p-3)^{n} p_{3}+\ldots,
$$

$p_{1}, p_{2}$, etc. étant les coëfficients du binôme. 
52. Schlömilch, formule pour les nombres de Bernoulli.

Nous partirons ici de la formule connue

3. $\quad \operatorname{tang}\left(\frac{1}{4} \pi+\frac{1}{2} x\right)=\sec x+\operatorname{tang} x$

$=\boldsymbol{B}_{0}+\frac{\boldsymbol{B}_{2}}{1 \cdot 2} x^{2}+\frac{\boldsymbol{B}_{4}}{1 \cdot 2 \cdot 3 \cdot 4} x^{4}+\frac{\boldsymbol{B}_{6}}{1 \cdot 2 \cdots \cdot 6} x^{6}+\ldots$

$+\frac{2^{2}\left(2^{2}-1\right)}{1 \cdot 2} B_{1} x+\frac{2^{4}\left(2^{4}-1\right)}{1 \cdot 2 \cdot 3 \cdot 4} B_{3} x^{3}+\frac{2^{6}\left(2^{6}-1\right)}{1 \cdot 2 \cdots 6} B_{5} x^{5}+\ldots$

En observant que l'on a aussi

$$
\operatorname{tang}\left(\frac{1}{4} \pi+\frac{1}{2} x\right)=\frac{1+\operatorname{tang} \frac{1}{2} x}{1-\operatorname{tang} \frac{1}{2} x}=\frac{\cos \frac{1}{2} x+\sin \frac{1}{2} x}{\cos \frac{1}{2} x-\sin \frac{1}{2} x}
$$

on obtient, en remplacant $x$ par $x /-1$,

$$
\begin{aligned}
\operatorname{tang}\left(\frac{1}{4} \pi+\frac{1}{2} x_{1}-1\right) & =\frac{e^{\frac{1}{2} x}+e^{-\frac{1}{2} x}+\left(e^{\frac{1}{2} x}-e^{-\frac{1}{2} \cdot x}\right)}{e^{\frac{1}{2} x}+e^{-\frac{1}{2} x}-\left(e^{\frac{1}{2} x}-e^{-\frac{1}{2} x}\right)} \frac{\sqrt{ }-1}{\sqrt{2}} \\
& =\frac{e^{x}+1+\left(e^{x}-1\right) \sqrt{ }-1}{e^{x}+1-\left(e^{x}-1\right) \sqrt{ }-1}=\frac{(1+\sqrt{ }-1) e^{x}+1-\sqrt{ }-1}{(1-\sqrt{ }-1) e^{x}+1+\sqrt{-1}},
\end{aligned}
$$

et par conséquent en vertu de $\mathrm{N}^{\circ}$. (3):

4. $\frac{(1+\sqrt{ }-1) e^{x}+1-\sqrt{ }-1}{(1-\sqrt{-1}) e^{x}+1+\sqrt{ }-1}=B_{0}-\frac{B_{2}}{1 \cdot 2} x^{2}+\frac{B_{4}}{1 \cdot 2 \cdot 3 \cdot 4} x^{4}-\ldots$

$$
+\sqrt{ }-1\left\{\frac{2^{2}\left(2^{2}-1\right)}{1 \cdot 2} B_{1} x-\frac{2^{4}\left(2^{4}-1\right)}{1 \cdot 2 \cdot 3 \cdot 4} B_{3} x^{3}+\ldots\right\} \text {. }
$$

D'un autre côté on a suivant le théorème de Mac-Laurin :

$$
\text { 5. } f(x)=f(0)+\frac{f^{\prime}(0)}{1} x+\frac{f^{\prime \prime}(0)}{1 \cdot 2} x^{2}+\frac{f^{\prime \prime \prime}(0)}{1 \cdot 2 \cdot 3} x^{3}+\ldots
$$

tant que cette série est convergente, et si l'on suppose

$$
\text { 6. } f x=\frac{(1+\sqrt{ }-1) e^{x}+1-\sqrt{-1}}{(1-\sqrt{-1}) e^{x}+1+\sqrt{-1}},
$$

on obtient, en comparant les équations ( 4 et 5 ):

$$
\begin{aligned}
\frac{f^{(n)}(0)}{1 \cdot 2 \cdots n} & =(-1)^{3 n} \frac{B_{n}}{1 \cdot 2 \cdots \cdot n}, \text { si } n \text { est pair, } \\
\frac{f^{(n)}(0)}{1 \cdot 2 \cdots \cdot n} & =(-1)^{\frac{1}{(n-1)}} \frac{2^{n+1}\left(2^{n+1}-1\right)}{1 \cdot 2 \cdot 3 \cdots(n+1)} B_{n} V-1 \text {, si } n \text { est impair; }
\end{aligned}
$$

ou bien

7. $f^{(n)}(0)=(-1)^{\frac{1 n}{2}} \boldsymbol{B}_{n}$ pour des valeurs paires de $n$, et

8. $f^{(n)}(0)=(-1)^{\frac{1}{(n-1)}} \cdot \frac{2^{n+1}\left(2^{n+1}-1\right)}{n+1} B_{n} \sqrt{ }-1$ pour des valeurs impaires de $n$.

Crelle's Journal f. d. M. Ba. XXXII. Heft 4. 
Or à l'aide de la formule (1) il est facile de trouver la dérivée à gauche. En effet, en supposant plus généralement:

$$
f(z)=\frac{\alpha z+\beta}{\beta z+\alpha}=\frac{1}{\beta}\left\{\alpha+\frac{\beta^{2}-\alpha^{2}}{\beta z+\alpha}\right\}
$$

on aura

$$
\begin{aligned}
f^{(p)}(z) & =(-1)^{p} 1 \cdot 2 \cdots p \cdot \frac{\beta^{2}-\alpha^{2}}{\beta(\beta z+\alpha)}\left(\frac{\beta}{\beta z+\alpha}\right)^{p} \text { et } \\
\frac{1}{1 \cdot 2 \cdots p} f^{(p)}\left(e^{x}\right) & =\frac{(-1)^{p}}{\beta} \cdot \frac{\beta^{2}-\alpha^{2}}{\beta e^{x}+\alpha}\left(\frac{\beta}{\beta c^{x}+\alpha}\right)^{p} .
\end{aligned}
$$

Substituant ces valeurs dans l'équation (1), on obtient

$$
\begin{gathered}
\frac{\partial^{n}}{\partial x^{n}}\left(\frac{\alpha e^{x}+\beta}{\beta e^{x}+\alpha}\right) \\
=\frac{\alpha^{2}-\beta^{2}}{\beta\left(\beta e^{x}+\alpha\right.}\left\{K_{1}^{n}\left(\frac{\beta e^{x}}{\beta e^{x}+\alpha}\right)-\mathscr{K}_{2}\left(\frac{\beta e^{x}}{\beta e^{x}+\alpha}\right)^{2}+\stackrel{n}{K}_{3}\left(\frac{\beta e^{x}}{\beta e^{x}+\alpha}\right)^{3}-\ldots\right\},
\end{gathered}
$$

et en posant maintenant $x=0$, on trouve que

$$
\text { 9. } f(x)=\frac{\alpha e^{x}+\beta}{\beta e^{x}+\alpha}
$$

donne

$$
\text { 10. } f^{(n)}(0)=\frac{\alpha-\beta}{\beta}\left\{\boldsymbol{K}_{1}^{n}\left(\frac{\beta}{\alpha+\beta}\right)-\stackrel{n}{\boldsymbol{K}}_{2}\left(\frac{\beta}{\alpha+\beta}\right)^{2}+\stackrel{n}{\boldsymbol{K}}_{3}\left(\frac{\beta}{\alpha+\beta}\right)^{3}-\ldots\right\} .
$$

La valeur de $f(x)$ dans (6) n'est qu'un cas particulier de (9); savoir celui que donnent les valeurs $\alpha=1+\gamma-1$ et $\beta=1-\sqrt{-1}$ de $\alpha$ et $\beta$. Donc on aura, reduction faite :

$$
f^{(n)}(0)=\sqrt{ }-1\left\{\stackrel{n}{K}_{1}-\stackrel{n}{K}_{2, \frac{1}{2}}(1-\sqrt{ }-1)+\stackrel{n}{K}_{3} \frac{1}{2}(1-\sqrt{ }-1)^{2}-\ldots . .\right.
$$

Pour décomposer cette série en parties réelles et imaginaires, il suffit d'observer que $1-\sqrt{ }-1=\left(\cos \frac{1}{4} \pi-\sqrt{ }-1 \sin \frac{1}{4} \pi\right) \sqrt{ } 2$ et d'appliquer à chaque terme le théoréme de Moivre. Cela donne

$$
\begin{aligned}
& f^{(n)}(0)=-\frac{1}{(\sqrt{ } 2)} \stackrel{n}{K}_{2} \sin \frac{1}{4} \pi+\frac{1}{\left(\gamma^{2}\right)^{2}} \stackrel{n}{K}_{3} \sin _{\frac{2}{4}} \pi-\frac{1}{(\sqrt{ } 2)^{3}} \stackrel{n}{K}_{4} \sin _{4}^{3} \pi+\ldots \\
& :+\sqrt{ }-1\left\{\stackrel{n}{K}_{1}-\frac{1}{\sqrt{ } 2} \stackrel{n}{K}_{2} \cos \frac{1}{4} \pi+\frac{1}{(\sqrt{ } 2)^{2}} \ddot{K}_{3} \cos \frac{2}{4} \pi-\frac{1}{(\sqrt{ } 2)^{3}} \stackrel{n}{K}_{4} \cos \frac{3}{4} \pi+\ldots\right\} .
\end{aligned}
$$


La comparaison de cette équation avec les formules ( 7 et 8 ) conduit aux équations suivantes:

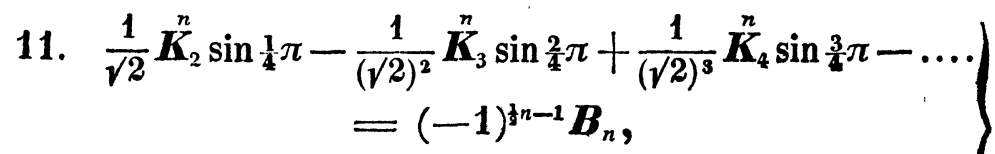
pour $\boldsymbol{n}$ pair;

12. $\tilde{K}_{1}-\frac{1}{\sqrt{2}} \stackrel{n}{K}_{2} \cos \frac{1}{4} \pi+\frac{1}{(\sqrt{ } 2)^{2}} \stackrel{n}{K}_{3} \cos \frac{2}{4} \pi-\ldots=0$,

13. $\quad \stackrel{n}{K}_{1}-\frac{1}{\sqrt{2}} \stackrel{n}{K_{2}} \cos \frac{1}{4} \pi+\frac{1}{(\sqrt{ } 2)^{2}} \stackrel{n}{K}_{3} \cos \frac{2}{4} \pi-\ldots$

$$
=(-1)^{\frac{2}{2(n-1)}} \cdot \frac{2^{n+1}\left(2^{n+1}-1\right)}{n+1} B_{n}
$$
pour un $n$ impair.

14. $\frac{1}{\sqrt{ } 2} K_{2}^{n} \sin \frac{1}{4} \pi-\frac{1}{(\sqrt{ } 2)^{2}} K_{3}^{n} \sin \frac{2}{4} \pi+\frac{1}{(\sqrt{ } 2)^{3}} K_{4}^{n} \sin _{\frac{3}{4}} \pi-\ldots=0$,

Il est facile aussi de réunir les deux équations (11 et 13) dans une seule. En effet les quantités

$$
(-1)^{\frac{1}{2}(n-2)} B_{n} \quad \text { et } \quad(-1)^{\frac{1}{3}(n-1)} \cdot \frac{2^{n+1}\left(2^{n+1}-1\right)}{n+1} B_{n}
$$

peuvent être exprimées toutes les deux en même temps par

$$
(-1)^{\frac{1}{2}(n-1-\mu)} \frac{2^{\lambda(n+1)+\mu}\left(2^{\lambda(n+1)+\mu}-1\right)}{\lambda n+\mu+1} B_{n},
$$

en prenant les quantités $\lambda$ et $\mu$ telles, que pour des valeurs paires de $n$ :

$$
\lambda=0 \text { et } \mu=1 \text {, }
$$

et pour des valeurs impaires de $n$ :

$$
\lambda=1 \text { et } \mu=0 \text {. }
$$

On satisfera à ces conditions en posant

$$
\text { 15. } \lambda=\frac{1}{2}\left(1-(-1)^{n}\right) \text { et } \mu=\frac{1}{2}\left(1-(-1)^{n-1}\right) \text {. }
$$

Alors les équations (11 et 13) peuvent être exprimées par

$$
\begin{gathered}
(-1)^{\frac{1}{(2(n-1-\mu)}} \frac{2^{\lambda(n+1)+\mu}\left(2^{\lambda(n+1)+\mu}-1\right)}{\lambda n+\mu+1} B_{n} \\
=\lambda\left\{K_{1}^{n}-\frac{1}{\sqrt{2}} \stackrel{n}{K}_{2} \cos \frac{1}{4} \pi+\frac{1}{(\sqrt{ } 2)^{2}} \stackrel{n}{K}_{3} \cos \frac{2}{4} \pi-\frac{1}{(\sqrt{2})^{3}} \stackrel{n}{K}_{4} \cos \frac{3}{4} \pi+\ldots\right\} \\
+\mu\left\{\frac{1}{\sqrt{ } 2} \stackrel{n}{K}_{2} \sin \frac{1}{4} \pi-\frac{1}{(\sqrt{ } 2)^{2}} \stackrel{n}{K}_{3} \sin \frac{2}{4} \pi+\frac{1}{(\sqrt{2})^{3}} \stackrel{n}{4}_{4} \sin \frac{3}{4} \pi-\ldots\right\}
\end{gathered}
$$


ou bien par

$$
\begin{gathered}
\text { 16. } \quad(-1)^{\frac{3(n-1-\mu)}{2(n+1)+\mu}\left(2^{2(n+1)+\mu}-1\right)} \frac{2^{\lambda(n+\mu+1}}{\lambda n+\mu+1} B_{n} \\
=\sum_{x=1}^{i=n}(-1)^{r-1} K_{r}^{n} \frac{\lambda \cos \frac{1}{4}((r-1) \pi)-\mu \sin \frac{1}{4}((r-1) \pi)}{\frac{1}{2}(r-1)} .
\end{gathered}
$$

Voilà la formule dont nous nous sommes proposé le développement. Jena, mai 1846. 


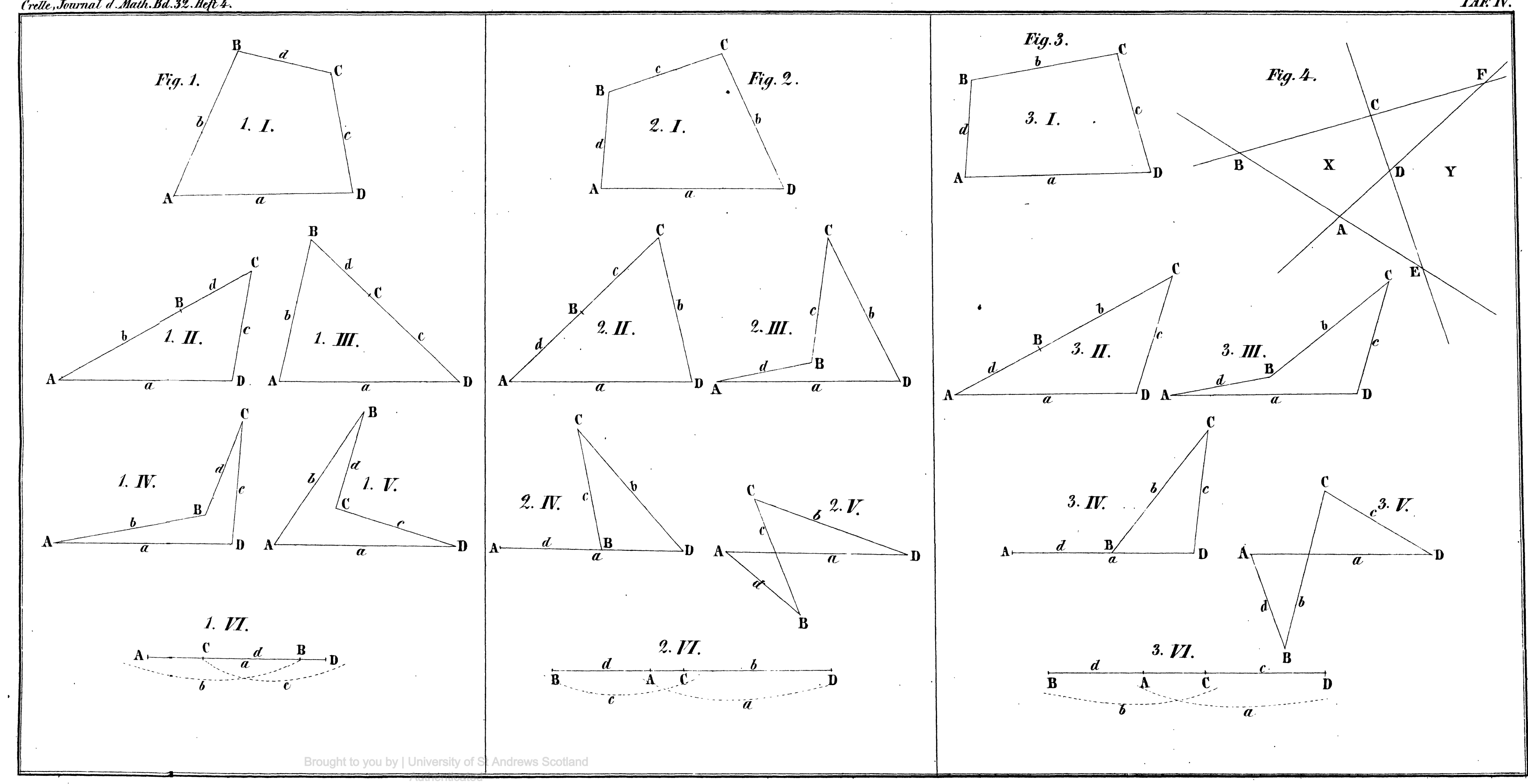


Brought to you by | University of St Andrews Scotland Authenticated

Download Date | 6/16/15 10:29 AM 
Crelle, Toumal d. Math:Bd:8XXII Heft4.

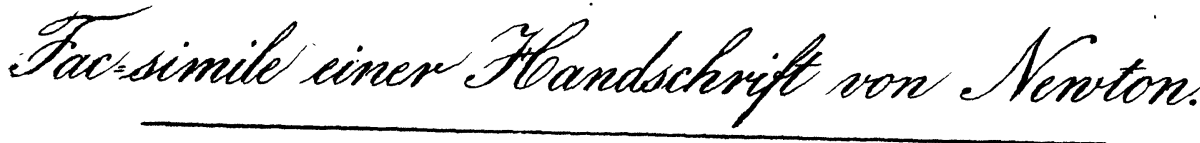

Godrfrido Gulielmo Sribnitio fracus Nuwton S.P.D.

Lilera he, lim non stakin aceyply respontarun, e maniby clapse inkr sathdas mras Jive lakeers, are in eas ank

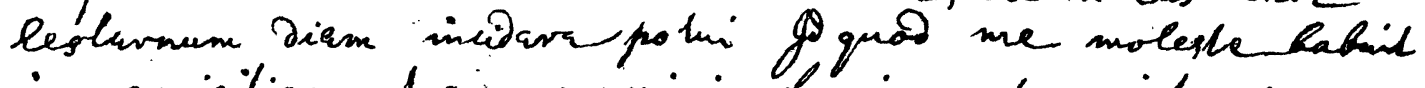
cim ansieiliam hain maximi faciam, $k g / 3$ inthr summos hujus socenti Gsometras a multis ratro annis haburnin;

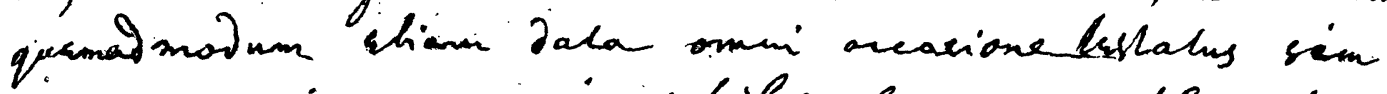
nan gramini commircia phibosophica omathrmatiza quanmaximi fugiam, tamen maturbam ne jikeatia amieitia nostrat axe shlentio Lecramentum aceifurvaly itys

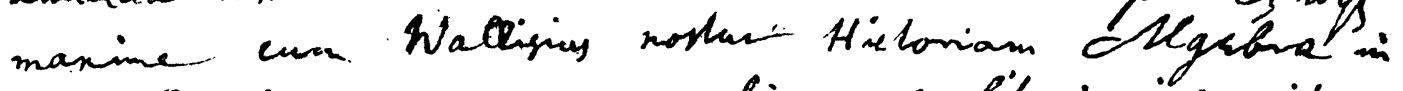

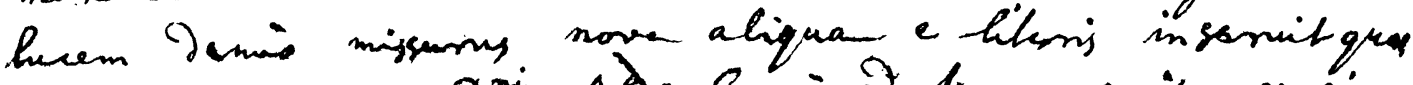

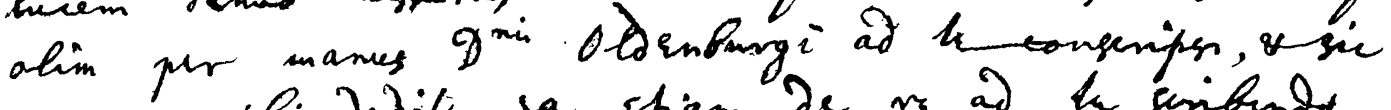
ensam mith Jabit sa cham be re ad he seribunt. Postulavid sinin al melhodum quanam Dupticem ape.

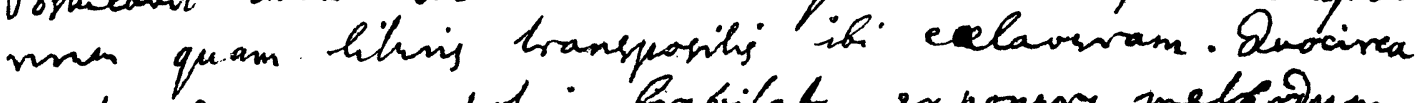

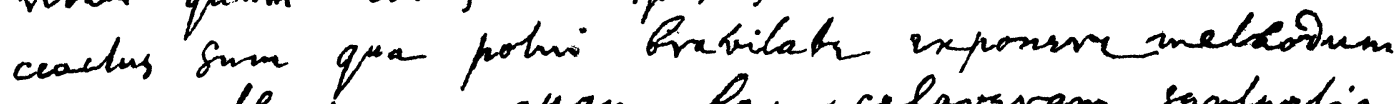
maam fhrionm quam hac celavaram santurtia. Dala aqualione quantitals qualenng fluenty sivolusn

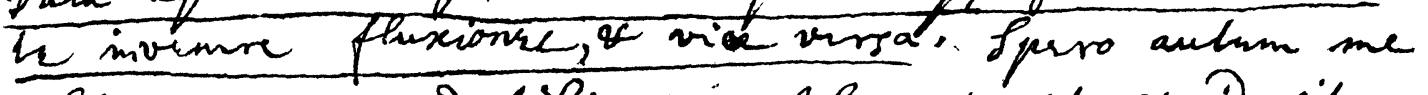
nibil scripsuss quod liki mon placeal, el squid sil quod vapuekensione Digmum censkas. nt htersy id miki rigmifices quoniam annicos phessis facio quam inventa mallermalica.

Lasi vahi me libi amieuen intugeminam esse oc ainitition ham maximi. facere. Vale DaPam Cantabrigie, 0 clob. $\frac{16}{26} \cdot 1693$. 